\title{
Electronic wavefunction with maximally entangled MPS representation
}

\author{
Benedikt R. Graswald ${ }^{\mathrm{a}}$ (D) and Gero Friesecke ${ }^{\mathrm{b}}$ \\ Department of Mathematics, Technical University of Munich, Munich, Germany
}

Received 7 April 2021 / Accepted 28 May 2021 / Published online 10 June 2021

(C) The Author(s) 2021

\begin{abstract}
We present an example of an electronic wavefunction with maximally entangled MPS representation, in the sense that the bond dimension is maximal and cannot be lowered by any re-ordering of the underlying one-body basis. Our construction works for any number of electrons and orbitals.
\end{abstract}

\section{Introduction}

It has long been recognized that matrix product states (MPS) yield accurate representations of quantum chemical wavefunctions. Such representations lie at the heart of the QC-DMRG method, a state-of-the-art method for strongly correlated systems [1-5]. However, exactness requires exponentially large matrices with respect to the system size and the quality of the approximation is governed by the size of the discarded singular values of the corresponding unfoldings $\psi_{\mu_{k}+1, \ldots, \mu_{L}}^{\mu_{1}, \ldots, \mu_{k}}[5,6]$.

Unlike in spin chains with identical sites, where the required matrix sizes are connected to the entanglement between subsystems which is in turn governed by area laws [7-10], the situation in quantum chemistry is more complicated. The role of the sites is then taken by the system's molecular orbitals, and the matrix ranks, the singular values, and the overall approximation quality is strongly influenced by the ordering of the orbitals $[3,11-$ 14]. Reordering the orbitals corresponds to changing the topology of the tensor network underlying the MPS; see Fig. 1. As turns out, standard examples with maximal entanglement such as the fermionic Bell states (see below) have the feature that the largest matrix rank (or bond dimension) for $L$ molecular orbitals occupied by $N=L / 2$ electrons drops from maximal, $2^{L / 2}$, to just 2 independently of $L$, under optimal re-ordering.

Here we present an explicit, rather more intricately correlated state whose bond dimension stays at the maximal value $2^{L / 2}$, regardless of any re-ordering.

\section{MPS representation}

Given a suitable orthonormal set $\left\{\varphi_{1}, \ldots, \varphi_{L}\right\}$ of molecular spin orbitals, typically consisting of occupied and

\footnotetext{
a e-mail: benedikt.graswald@ma.tum.de (corresponding author)

b e-mail: gf@ma.tum.de
}

unoccupied Hartree-Fock orbitals, recall the exponentialsized full-CI expansion of an electronic wavefunction which reads, in $N$-particle, respectively, Fock space,

$$
\begin{aligned}
\Psi & =\sum_{i_{1}<\ldots<i_{N}} \lambda_{i_{1}, \ldots, i_{N}}\left|\varphi_{i_{1}}, \ldots, \varphi_{i_{N}}\right\rangle \\
& =\sum_{\mu_{1}, \ldots, \mu_{L}=0}^{1} \psi_{\mu_{1}, \ldots, \mu_{L}} \Phi_{\mu_{1}, \ldots, \mu_{L}} .
\end{aligned}
$$

Here the $\left|\varphi_{i_{1}}, \ldots, \varphi_{i_{N}}\right\rangle$ are Slater determinants and

$$
\begin{aligned}
\psi_{\mu_{1}, \ldots, \mu_{L}} & = \begin{cases}\lambda_{i_{1}, \ldots, i_{N}}, & \text { if } \mu_{i_{1}}=\ldots=\mu_{i_{N}}=1, \sum_{j} \mu_{j}=N \\
0, & \text { else, }\end{cases} \\
\Phi_{\mu_{1}, \ldots, \mu_{L}} & = \begin{cases}\left|\varphi_{i_{1}} \ldots, \varphi_{i_{N}}\right\rangle, & \text { if } \mu_{i_{1}}=\ldots=\mu_{i_{N}}=1 \\
0, & \text { else. }\end{cases}
\end{aligned}
$$

The MPS approximation consists in the ansatz

$$
\psi_{\mu_{1}, \ldots, \mu_{L}}=A_{1}\left[\mu_{1}\right] \cdots A_{L}\left[\mu_{L}\right]
$$

where the $A_{k}\left[\mu_{k}\right]$ are matrices of size $1 \times M$ (for $k=1$ ), $M \times M($ for $k=2, \ldots, L-1)$, and $M \times 1($ for $k=L)$ for some moderate value of $M$.

\section{Fermionic Bell states}

Next, we argue that prototype examples of strong entanglement from spin physics and QIT-like Bell states - are in fact only weakly entangled in the MPS sense if re-ordering of the "sites" is allowed. Of course re-ordering only makes sense for molecular orbitals, not sites in 1D spin chains. 


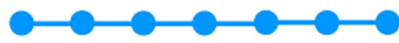

(a) Original MPS

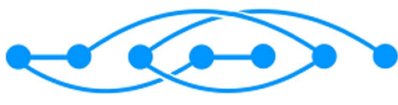

(b) Reordered MPS

Fig. 1 Schematic picture of a MPS before and after reordering the orbitals (vertices). Bonds represent virtual variables, i.e., summation indices in the matrix product; see Eq. (3)

For $N$ electrons occupying $L=2 N$ orbitals $\left\{\varphi_{1}, \ldots\right.$, $\left.\varphi_{L}\right\}$, one can easily write down a fermionic analogon to the standard Bell states.

Set $\psi_{k}:=\left(\varphi_{k}+\varphi_{k+N}\right) / \sqrt{2}$ for $k=1, \ldots, N$ and consider the Slater determinant $\Psi:=\left|\psi_{1}, \ldots, \psi_{N}\right\rangle$. It is then not hard to see (e.g., [15]) that its minimal MPS representation in the basis $\left(\varphi_{k}\right)_{k}$ has bond dimension $2^{N}$.

Now apply a re-ordering which puts paired-up orbitals next to each other,

$$
\left(\tilde{\varphi}_{1}, \tilde{\varphi}_{2}, \ldots, \tilde{\varphi}_{L-1}, \tilde{\varphi}_{L}\right)=\left(\varphi_{1}, \varphi_{N+1}, \ldots, \varphi_{N}, \varphi_{L}\right) .
$$

We claim that in the new basis $\left(\tilde{\varphi}_{k}\right)_{k}, \Psi$ has an MPS representation with bond dimension just 2 . Indeed

$$
\Psi=\sum_{\mu_{1}, \ldots, \mu_{L}=0}^{1} A_{1}\left[\mu_{1}\right] \cdots A_{L}\left[\mu_{L}\right] \tilde{\Phi}_{\mu_{1}, \ldots, \mu_{L}}
$$

where $\tilde{\Phi}$ is specified as in (2) and the matrices $A_{k}$ are

$$
\begin{aligned}
A_{1}\left[\mu_{1}\right] & =\left(\begin{array}{cc}
\delta_{\mu_{1}}^{0} & \delta_{\mu_{1}}^{1}
\end{array}\right), A_{L}\left[\mu_{L}\right]=\left(\delta_{\mu_{1}}^{1}, \delta_{\mu_{1}}^{0}\right)^{T}, \\
A_{2 \ell}\left[\mu_{2 \ell}\right] & =\left(\begin{array}{cc}
\delta_{\mu_{2 \ell}}^{1} & 0 \\
0 & \delta_{\mu_{2 \ell}}^{0}
\end{array}\right), A_{2 \ell+1}\left[\mu_{2 \ell+1}\right]=\left(\begin{array}{ll}
\delta_{\mu_{2 \ell+1}}^{0} & \delta_{\mu_{2 \ell+1}}^{1} \\
\delta_{\mu_{2 \ell+1}}^{0} & \delta_{\mu_{2 \ell+1}}^{1}
\end{array}\right) .
\end{aligned}
$$

Here $\ell=1, \ldots, N-1$ and $\delta_{\nu}^{k}$ denotes the Kronecker delta.

\section{Maximally entangled state}

To construct a state whose bond dimension cannot be reduced by any re-ordering, we start off by recalling an old result by Besicovitch [16]; let $p_{1}, \ldots p_{s}$, be different primes. Then

Theorem 1 (Corollary 1 in [16]) A polynomial $P\left(\sqrt{p_{1}}\right.$, $\left.\ldots, \sqrt{p_{s}}\right)$ with rational coefficients and degree w.r.t. each entry less than or equal to 1, not all equal to zero, cannot vanish.

Now we consider the set $\mathcal{P}:=\left\{\sqrt{p_{j}}: p_{j}\right.$ prime $\}$. Then every matrix $A$ whose elements belong to $\mathcal{P}$ and are pairwise different has maximal rank, since - for every square submatrix $B-\operatorname{det}(B)$ is exactly a polynomial of the above form.

Now define the state $\Psi_{\mathcal{P}}$ by

$$
\begin{aligned}
\Psi_{\mathcal{P}} & =\sum_{i_{1}<\ldots<i_{N}} \lambda_{i_{1}, \ldots, i_{N}}\left|\varphi_{i_{1}}, \ldots, \varphi_{i_{N}}\right\rangle \\
& =\sum_{\mu_{1}, \ldots, \mu_{L}=0}^{1} \psi_{\mu_{1}, \ldots, \mu_{L}} \Phi_{\mu_{1}, \ldots, \mu_{L}}
\end{aligned}
$$

where the coefficients $\lambda_{i_{1}, \ldots, i_{N}}$ are mutually different elements of $\mathcal{P}$ and the second equation gives the occupation representation with $\psi_{\mu_{1}, \ldots, \mu_{L}}$ corresponding to $\lambda_{i_{1}, \ldots, i_{N}}$ as in (2). Then every unfolding $\psi_{\mu_{k+1}, \ldots, \mu_{L}}^{\mu_{1}, \ldots, \mu_{k}}$ is a matrix of the above form and thus has maximal rank. In particular [17], $\Psi$ has maximal bond dimension.

Furthermore if we consider any new ordering, that is, we change our orbitals according to $\left(\varphi_{1}, \ldots, \varphi_{L}\right)=$ $Q\left(\tilde{\varphi}_{1}, \ldots, \tilde{\varphi}_{L}\right)$ with $Q \in \mathbb{R}^{L \times L}$ a permutation matrix, then we cannot decrease the rank of any unfolding. Indeed, it is easy to see that we then obtain the following representation:

$$
\begin{aligned}
\Psi_{\mathcal{P}} & =\sum_{j_{1}<\ldots<j_{N}} \tilde{\lambda}_{j_{1}, \ldots, j_{N}}\left|\tilde{\varphi}_{j_{1}}, \ldots, \tilde{\varphi}_{j_{N}}\right\rangle \\
& =\sum_{\mu_{1}, \ldots, \mu_{L}=0}^{1} \tilde{\psi}_{\mu_{1}, \ldots, \mu_{L}} \tilde{\Phi}_{\mu_{1}, \ldots, \mu_{L}},
\end{aligned}
$$

with

$$
\tilde{\lambda}_{j_{1}, \ldots, j_{N}}=\sum_{i_{1}<\ldots<i_{N}} \lambda_{i_{1}, \ldots, i_{N}}\left|\begin{array}{ccc}
q_{i_{1}, j_{1}} & \ldots & q_{i_{1}, j_{N}} \\
\vdots & & \vdots \\
q_{i_{N}, j_{1}} & \ldots & q_{i_{N}, j_{N}}
\end{array}\right|
$$

where $q_{i j}$ denotes the elements of $Q$. Since $Q$ is a permutation, exactly one determinant will be non-zero. Thus every unfolding still contains the same elements but only their positions change. But by construction of the set $\mathcal{P}$, the position within the unfolding $\tilde{\psi}_{\mu_{k+1}, \ldots, \mu_{L}}^{\mu_{1}, \ldots, \mu_{k}}$ is irrelevant as long as all entries are different elements of $\mathcal{P}$. Hence the unfolding still has full rank. Therefore $\Psi_{\mathcal{P}}$ still has maximal bond dimension.

We remark that in contrast to orderings, arbitrary fermionic mode transformations, i.e., choosing the transformation $Q$ above as a unitary, can always somewhat decrease the bond dimension. In the two-particle case $(N=2)$, this can even achieve the optimal bond dimension of 3 , for an arbitrary number of orbitals $L$ [18].

\section{Singular value distribution}

We have also numerically calculated the singular value distribution of our example states for different values of $N$ and $L$ and different orderings (such as the widely 


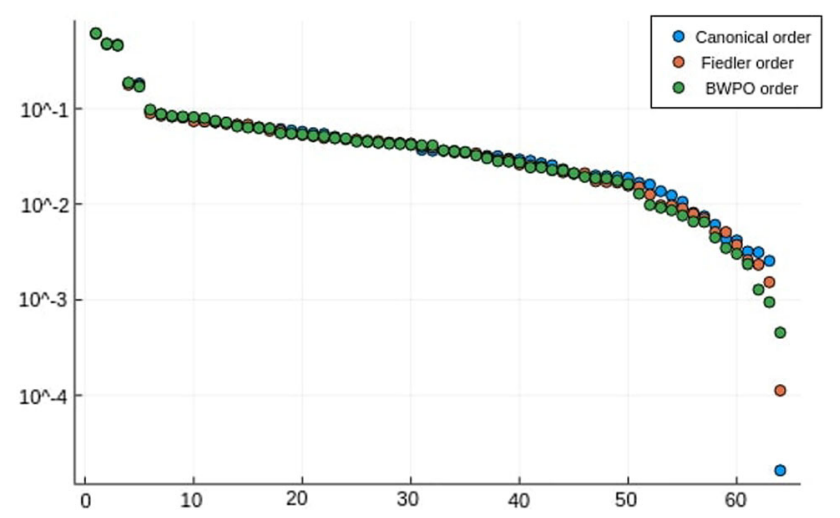

Fig. 2 Singular value distribution of the matrization $\psi_{\mu_{7}, \ldots, \mu_{12}}^{\mu_{1}, \ldots, \mu_{6}}$ of the state $\Psi_{\mathcal{P}}[\mathrm{Eq} .(5)]$ with $N=6$ electrons and $L=12$ orbitals, for different orderings

used Fiedler order [11]) using the code tensor-train-julia [19].

Figure 2 corresponds to $N=6, L=12$, and a random choice of $\left(\begin{array}{l}L \\ N\end{array}\right)$ primes of size less than $2^{N+L}$. The different orderings shown are the original (canonical) order, the Fiedler order [11], and the more recent best weighted prefactor order [15]. In particular all $2^{L / 2}$ singular values are non-zero, as predicted.

The singular values are seen to decay extremely slowly, and exhibit a remarkable almost-invariance under re-ordering. A less extreme but related observation, that the bond dimension cannot be lowered much by re-ordering, was made in an interesting numerical study of strongly correlated states in the 1D Hubbard model [20]. By contrast, for weakly correlated states re-ordering typically reduces the tail by several order of magnitude [15].

Physically, the slow decay in Fig. 2 means that for the state $\Psi_{\mathcal{P}}$, any two subsystems obtained by partitioning the molecular orbitals into two equal-size parts are strongly entangled.

Acknowledgements The authors thank M.-S. Dupuy for helpful discussions. Support from the International Research Training Group IGDK Munich - Graz funded by DFG, Project Number 188264188/GRK1754, is gratefully acknowledged.

Funding Open Access funding enabled and organized by Projekt DEAL.

Data Availability Statement This manuscript has no associated data or the data will not be deposited. [Authors' comment: In Figure 2 we took a random choice of $L$ choose $\mathrm{N}$ random primes of size less than $2^{(N+L)}$. The shape of the plot and all the implications drawn in the paper do not depend on this particular choice. We always obtained the same overall pattern for any random selection of primes. Even averaging over thousands of different samples did not change the profile of the plot. So, since Fig. 2 just provides a generic example and is in no way special, there is no scientific data to provide.]
Open Access This article is licensed under a Creative Commons Attribution 4.0 International License, which permits use, sharing, adaptation, distribution and reproduction in any medium or format, as long as you give appropriate credit to the original author(s) and the source, provide a link to the Creative Commons licence, and indicate if changes were made. The images or other third party material in this article are included in the article's Creative Commons licence, unless indicated otherwise in a credit line to the material. If material is not included in the article's Creative Commons licence and your intended use is not permitted by statutory regulation or exceeds the permitted use, you will need to obtain permission directly from the copyright holder. To view a copy of this licence, visit http://creativecomm ons.org/licenses/by/4.0/.

\section{References}

1. S.R. White, R.L. Martin, Ab initio quantum chemistry using the density matrix renormalization group. J. Chem. Phys. 110, 4127 (1999)

2. H.-G. Luo, M.-P. Qin, T. Xiang, Optimizing hartreefock orbitals by the density-matrix renormalization group. Phys. Rev. B 81, 235129 (2010)

3. O. Legeza, J. Röder, B.A. Hess, Controlling the accuracy of the density-matrix renormalization-group method: the dynamical block state selection approach. Phys. Rev. B 67, 125114 (2003a)

4. S. Szalay, M. Pfeffer, V. Murg, G. Barcza, F. Verstraete, R. Schneider, O. Legeza, Tensor product methods and entanglement optimization for ab initio quantum chemistry. Int. J. Quantum Chem. 115, 1342 (2015)

5. U. Schollwöck, The density-matrix renormalization group. Rev. Mod. Phys. 77, 259 (2005)

6. W. Hackbusch, Tensor Spaces and Numerical Tensor Calculus, vol. 42 (Springer, Berlin, 2012)

7. M.B. Plenio, J. Eisert, J. Dreißig, M. Cramer, Entropy, entanglement, and area: analytical results for harmonic lattice systems. Phys. Rev. Lett. 94, 060503 (2005)

8. J. Eisert, M. Cramer, M.B. Plenio, Colloquium: area laws for the entanglement entropy. Rev. Mod. Phys. 82, 277 (2010)

9. K. Van Acoleyen, M. Mariën, F. Verstraete, Entanglement rates and area laws. Phys. Rev. Lett. 111, 170501 (2013)

10. M.B. Hastings, An area law for one-dimensional quantum systems. J. Stat. Mech Theory Exp. 2007, P08024 (2007)

11. G. Barcza, O. Legeza, K.H. Marti, M. Reiher, Quantuminformation analysis of electronic states of different molecular structures. Phys. Rev. A 83, 012508 (2011)

12. K. Boguslawski, P. Tecmer, O. Legeza, M. Reiher, Entanglement measures for single- and multireference correlation effects. J. Phys. Chem. Lett. 3, 3129 (2012)

13. O. Legeza, J. Sólyom, Optimizing the density-matrix renormalization group method using quantum information entropy. Phys. Rev. B 68, 195116 (2003)

14. O. Legeza, J. Röder, B.A. Hess, QC-DMRG study of the ionic-neutral curve crossing of LiF. Mol. Phys. 101, 2019 (2003b) 
15. M.-S. Dupuy, G. Friesecke, Inversion symmetry of singular values and a new orbital ordering method in tensor train approximations for quantum chemistry. SIAM J. Sci. Comput. 43, B108 (2021)

16. A.S. Besicovitch, On the linear independence of fractional powers of integers. J. Lond. Math. Soc. 1, 3 (1940)

17. S. Holtz, T. Rohwedder, R. Schneider, On manifolds of tensors of fixed TT-rank. Numer. Math. 120, 701 (2012)
18. G. Friesecke, B. Graswald, Two-particle correlation in QC-DMRG, in preperation (2021)

19. M.-S. Dupuy, Tensor-train-julia (2021). https://github. com/msdupuy/Tensor-Train-Julia. Accessed 3 Apr 2021

20. Ö. Legeza, F. Gebhard, J. Rissler, Entanglement production by independent quantum channels. Phys. Rev. B 74, 195112 (2006) 